\title{
Rheumatic disorders and primary biliary cirrhosis: an appraisal of 170 Italian patients
}

\author{
B Marasini, M Gagetta, V Rossi, P Ferrari
}

\begin{abstract}
Objective-To establish the frequency of connective tissue diseases (CTD) in a cohort of Italian patients with primary biliary cirrhosis (PBC) and to evaluate the availability of a marker for the early identification of the more common associated CTD.

Methods-One hundred and seventy consecutive patients with histologically diagnosed PBC were screened for the presence of a CTD and/or Raynaud's phenomenon (RP). Patients were classified as having a CTD only if they fulfilled standardised criteria.

Results-Forty seven patients had a CTD. The most common CTD was systemic sclerosis (SSc), found in 21 patients. RP was present in 54 patients, most of whom $(n=39)$ had an associated CTD. The most prevalent autoantibodies included antinuclear antibodies (ANA) with anticentromere (ACA) and speckled patterns (34 and 33 patients, respectively) and extractable nuclear antigens (ENA, 27 patients). However, while the frequencies of ACA and ENA were significantly higher in patients with an associated CTD $(p<0.0001$ and $p<0.005$, respectively), no relationship was found for speckled ANA. ACA was the best predictor of a CTD in patients with PBC (odds ratio (OR) 24.5 , 95\% CI 5.5 to 108.8), followed by the presence of ENA (OR 23.9, 95\% CI 5.6 to 101.0$)$ and RP (OR 20.2, 95\% CI 5.7 to 71.2 ).

Conclusions-Using strict standardised classification criteria we have found that SSc is the most common CTD associated with PBC and that ACA and ENA are strong markers for an associated CTD in patients with PBC.

(Ann Rheum Dis 2001;60:1046-1049)
\end{abstract}

Medicine, Surgery and

Dentistry, S Paolo

Hospital, University of

Milan, Milan, Italy

B Marasini

V Rossi

Clinical Chemistry Laboratory, $S$ Paolo Hospital, Milan, Italy M Gagetta

Statistical Sciences, University of Milano-Bicocca, Milan, Italy

P Ferrari

Correspondence to:

Dr B Marasini, Ospedale S

Paolo, Via Di Rudinì 8 ,

20142 Milan, Italy

bianca.marasini@unimi.it

Accepted 9 April 2001

Primary biliary cirrhosis (PBC) is a chronic progressive cholestatic liver disease characterised by destruction of intrahepatic bile ducts causing fibrosis and eventually cirrhosis of the liver. The cause of this disease is unknown, although the immunological abnormalities and

Table 1 Prevalence of connective tissue disease (CTD) or Raynaud's phenomenon (RP) in 170 patients with primary biliary cirrhosis (PBC)

\begin{tabular}{lccl}
\hline & Patients & Women & $\begin{array}{l}\text { Mean (SD) } \\
\text { age (years) }\end{array}$ \\
\hline CTD- RP- & $108(63 \%)$ & $97(90 \%)$ & $58(12)$ \\
CTD+ RP- & $8(5 \%)$ & $8(100 \%)$ & $59(8)$ \\
CTD+ RP+ & $39(23 \%)$ & $37(95 \%)$ & $61(9)$ \\
CTD- RP+ & $15(9 \%)$ & $14(93 \%)$ & $56(11)$ \\
\hline
\end{tabular}

the strong female preponderance point to an autoimmune aetiology.

Association with rheumatic diseases is well known. ${ }^{2}$ The presence of an associated autoimmune disease has been suggested to be predictive of a poorer prognosis, ${ }^{34}$ probably because, owing to improved management and liver transplantation, only the most severe cases of PBC die primarily from PBC. Since the associated conditions may be an important cause of morbidity and mortality, their early recognition is therefore mandatory for proper management. On the other hand, studies of the co-occurrence of autoimmune diseases may contribute to the knowledge of the still poorly understood mechanisms behind them.

The reported incidence and prevalence rates of PBC associated rheumatic diseases have often been conflicting, mainly because of methodological problems including diagnostic criteria and ethnic differences. ${ }^{5-7}$

The aims of this study were to assess the frequency of a number of connective tissue diseases (CTD) in a large cohort of Italian patients with $\mathrm{PBC}$ and to evaluate whether it is possible to find early marker(s) indicative of the cooccurrence of PBC and CTD. Moreover, since standardised definitions of diseases are necessary to compare data across studies, we evaluated only patients who fulfilled well accepted classification and/or diagnostic criteria.

\section{Methods}

One hundred and seventy consecutive Italian patients (156 women, 14 men) of mean age 59 (range 30-84) with histologically confirmed $\mathrm{PBC}^{8}$ who attended our centre between 1995 and 1998 entered the study after giving informed consent. The majority $(90 \%)$ were from Northern Italy. Patients were classified as having systemic sclerosis (SSc), systemic lupus erythematosus (SLE), or rheumatoid arthritis (RA) if they fulfilled the American College of Rheumatology (ACR) accepted criteria. ${ }^{9-11}$ Patients with Sjögren's syndrome (SS) fulfilled the criteria of Vitali et al. ${ }^{12}$ Mixed connective tissue disease (MCTD) was diagnosed by the presence of the symptoms described by Sharp et al. ${ }^{13}$ The diagnosis of polymyositis (PM) was based on Bohan and Peter's criteria. ${ }^{14}$ Patients with features strongly suggestive of autoimmune rheumatic disease (Raynaud's phenomenon (RP), skin thickening limited to the fingers, finger oedema, serum anticentromere antibody (ACA), with no internal organ abnormalities), but not fulfilling the criteria for any disorder, were classified as undifferentiated CTD (UCTD). ${ }^{15}$ Patients were diagnosed as 
Table 2 Prevalence of systemic sclerosis (SSc), undifferentieted connective tissue disese (UCTD), Sjögren's syndrome (SS), rheumatoid arthritis (RA), systemic lupus erythematosus (SLE), mixed connective tissue disease (MCTD) and polymyositis (PM) in 170 patients with primary biliary cirrhosis

\begin{tabular}{llllllll}
\hline & $S S c$ & UCTD & $S S$ & $R A$ & SLE & MCTD & PM \\
\hline $\begin{array}{l}\text { No of patients } \\
\%\end{array}$ & 21 & 12 & 6 & 3 & 3 & 1 & 1 \\
& 12.3 & 7.1 & 3.5 & 1.8 & 1.8 & 0.6 & 0.6
\end{tabular}

Table 3 Results of the best fitting logistical model

\begin{tabular}{lllll}
\hline & & & \multicolumn{2}{c}{$95 \%$ confidence intervals } \\
\cline { 4 - 5 } Variable & Odds ratio & p Value & Lower & Upper \\
\hline ACA & 24.5 & 0.0001 & 5.5 & 108.8 \\
ENA & 23.9 & 0.0001 & 5.6 & 101.0 \\
RP & 20.2 & 0.0001 & 5.7 & 71.2 \\
ACA-ENA & 0.02 & 0.0112 & 0.0 & 0.4 \\
Criterion & Intercept only & Intercept & and covariates & $\chi^{2}$ for covariates \\
-2 logL score & 187.4 & 83.4 & & 104.1 with $4 \mathrm{df}(\mathrm{p}=0.0001)$ \\
& & & & 93.3 with $4 \mathrm{df}(\mathrm{p}=0.0001)$ \\
\hline
\end{tabular}

having primary RP if they had typical episodic ischaemic attacks in response to cold or emotional stimuli for at least 2 years without clinical or serological evidence of CTD.

Antinuclear antibodies (ANA) were assayed by indirect immunofluorescence with HEp-2 cells (Immunoconcepts, Sacramento, CA, USA). Antimitochondrial antibodies (AMA) were assayed by indirect immunofluorescence on rat liver, kidney, and stomach sections (The Binding Site, Birmingham, UK). AMA negativity was confirmed by immunoblotting analysis of the PBC specific serum autoantibody against the M2 component of the mitochondrial inner membrane, as previously described. ${ }^{16}$ The following ANA patterns were considered: homogeneous, speckled, centromere, nucleolar. Antibodies to extractable nuclear antigens (ENA), including RNP, SS-A, SS-B, Jo-1, anti-topoisomerase I (ATA), and $\mathrm{Sm}$, were detected by an ELISA method (Diamedix Corp, Miami, FL, USA) and confirmed by immunoblotting analysis (Euroimmun $\mathrm{GmbH}$, Gross Groenau, Germany).

\section{STATISTICAL ANALYSIS}

ANOVA followed by the Scheffé test was used to test differences between groups. The $\chi^{2}$ test was used for categorical data. Logistic regression models were used to predict a binary dependent variable from a set of independent variables. The results are expressed as odds ratios (ORs) with 95\% confidence intervals (CI).

Data are mean (SD) unless otherwise specified. $p$ Values of $<0.05$ were considered significant.

\section{Results}

Sixty two patients $(36.5 \%)$ had a CTD and/or RP. Table 1 shows the prevalence of CTD and/or RP and the sex and age distribution among the groups. The most common CTD was SSc (table 2). Fifteen patients with SSc had the limited form of the disease (lc-SSc) and six had the diffuse form (dc-SSc). Fifty four patients (51 women) had RP, most of whom (39 patients, $72 \%$ ) presented with an associated CTD (SSc $(n=21)$; UCTD $(n=12)$; SS $(n=4)$; SLE and PM $(n=1$ each $))$. Most patients with UCTD had features of SSc; all had RP and ACA, three had skin thickening limited to the fingers, and three had telangiectasia. No patient had visceral involvement typical of SSc, and none had three or more of the CREST syndrome features (three patients could have been classified as RS, three as RT, and six as R). RP appeared before (1-52 years, mean 19 years, $n=26)$ or after $(1-16$ years, mean 5 years, $n=23$ ) the diagnosis of PBC. In five patients PBC and RP occurred almost simultaneously.

One hundred and sixty three patients with PBC had autoantibodies. However, excluding AMA which is typical of PBC and was found in 164 patients, autoantibodies were observed in $56 \%$ of patients with PBC, particularly if a CTD co-existed (87\% PBC with CTD v 44\% PBC without CTD, p<0.0001). The most prevalent autoantibodies included ANA with anticentromere $(n=34)$ and speckled $(n=33)$ patterns and ENA $(n=27)$. The frequencies of ACA and ENA were significantly higher in patients with PBC and CTD (ACA: $82 \%$ PBC with CTD $v 18 \%$ PBC without CTD, p $<0.0001$; ENA: $69 \%$ v 30\%, p<0.005), while the presence of the speckled pattern was independent of any autoimmune rheumatic conditions $(37 \%$ v $63 \%, \mathrm{p}=0.4018)$. Most of the ACA positive patients $(n=27,79 \%)$ had $R P$, but RP was unrelated to the presence of ACA (27/54 with RP had ACA and 27 did not). ACA were particularly frequent in UCTD and in SSc, with no significant differences between lc-SSc or dc-SSc ( $n=9$ and $n=4$, respectively). The frequencies of ACA and ENA significantly differed between SSc and UCTD (ACA: $\mathrm{SSc}=13$ patients positive $v \mathrm{UCTD}=$ all patients positive, $\mathrm{p}<0.02$; ENA: $\mathrm{SSc}=7$ patients positive $v \mathrm{UCTD}=$ all patients negative, $\mathrm{p}<0.05)$. ENA were particularly frequent in patients with SS $(n=4$ out of 6$)$. SS-A, the most common ENA $(n=16)$, was unrelated to rheumatic conditions (seven patients had PBC with no associated conditions, seven had a CTD, two had an isolated RP). SS-B was found in four patients, and in three it coexisted with SS-A (two patients had SS, one with no CTD). ATA was more frequent in CTD (three patients had dc-SSc, one had RA, one had PM, and one had PBC without rheumatic diseases). RNP was found in eight patients, five of whom had CTD; none of the patients had Sm or Jo-1. The ANA homogeneous pattern, found in 11 patients, was unrelated to CTD.

No relationship was observed between AMA negativity $(n=6)$, the other autoantibodies, and the different rheumatic disorders.

A multivariate analysis found that ACA was the best marker of an associated rheumatic disease, followed by the presence of ENA and RP (table 3).

\section{Discussion}

This study confirms that a relatively large proportion of patients with PBC have an autoimmune rheumatic disease.

To avoid the effects of geographical factors which have a role in PBC as well as in autoimmune rheumatic diseases, ${ }^{17}$ we performed 
our study on a cohort of Italian patients sufficiently large to draw conclusions about the co-existence of CTD in a defined population. Furthermore, we included only those patients with PBC who fulfilled well defined diagnostic or classification criteria for rheumatic diseases, which made it easier to compare data across studies.

The most frequent autoimmune rheumatic disease we found in patients with PBC was SSc. The co-existence of PBC and SSc is well known with a frequency ranging from 3\% to $50 \% .{ }^{1257}$ Even if patients with a "scleroderma spectrum" disorder are excluded, and although epidemiological studies of the prevalence of SSc in an Italian population are lacking, the prevalence of SSc in PBC appears to be significantly greater than that found in the general population. ${ }^{19} 20$ This finding may suggest some common aetiological mechanism, and certain peculiar aspects of the immune system reported in patients with PBC associated SSc seem to confirm this. ${ }^{21} 22$ Whether PBC and SSc have a chronic graft-versus-host disease pathogenesis, ${ }^{23}{ }^{24}$ possibly related to the persistence of microchimeric fetal cells in the circulation, ${ }^{25}{ }^{26}$ has to be clarified. It is tempting to speculate that the co-existence of PBC and SSc may be a new distinct clinical syndrome.

Since the frequencies of SS, SLE, MCTD, and PM were similar to those reported in the general population, ${ }^{20}{ }^{27-29}$ the co-occurrence with PBC should be considered casual. The previously reported ${ }^{30}$ high prevalence of SS in patients with PBC has not been confirmed in this study, probably because different classification criteria were used. The frequency of RA, which is similar to that observed in the general population, ${ }^{31}$ appears particularly high if compared with that recently reported in an Italian epidemiological survey. ${ }^{32}$ The previously reported greater frequency of $\mathrm{RA}$ in $\mathrm{PBC}^{16}$ probably also reflects different classification criteria. In fact, patients with PBC often complain of articular symptoms which do not fulfil the ACR criteria for RA ("arthritis of PBC"). ${ }^{2}$

Further studies over time are necessary to understand the significance of the cooccurrence of UCTD and PBC and, in particular, to evaluate whether these patients are at risk of SSc. It would be interesting to ascertain whether ACA, found in all UCTD, might identify a subset of patients with PBC who are destined to develop SSc. It is worth noting that ACA have been associated with severe vascular disease in $\mathrm{SSc}^{33}$ and that microvascular abnormalities have been found in PBC. ${ }^{34}$

The high frequency of RP is probably non-casual, although the prevalence of RP is difficult to assess. ${ }^{35}$ The significance of RP in patients with PBC and the relationship between RP and ACA are difficult to understand since RP was unrelated to ACA but, although weaker, RP was also a good indicator of an associated CTD. Whether or not RP is the result of an endothelial dysfunction is an interesting question that has to be settled.

With the exception of ENA, the frequency of the other autoantibodies was not related to
CTD. The significance of ATA is intriguing since half of the ATA positive patients with PBC did not have SSc.

In conclusion, using strict standardised classification and diagnostic criteria, we have found that a number of Italian patients with PBC have autoimmune rheumatic diseases, with SSc being the most common. Since autoimmune diseases constitute a leading cause of death among women, ${ }^{36}$ ACA and ENA, which are strongly indicative of a coexisting CTD, could be a useful prognostic test for patients with PBC. Further studies are needed to assess whether the combined occurrence of these diseases may provide new insight into their aetiopathogenesis.

1 Sherlock S, Schever PJ. The presentation and diagnosis of 100 patients with primary biliary cirrhosis. N Engl J Med 1973;289:674-8.

2 Culp KS, Fleming CR, Duffy J, Baldus WP, Dickson ER. Autoimmune associations in primary biliary cirrhosis Mayo Clin Proc 1982;57:365-70

3 Beswick DR, Klatskin G, Boyer JL. Asymptomatic primary biliary cirrhosis: a progress report on long term follow-up and natural history. Gastroenterology 1985;89:267-71.

4 Nyberg A, Loof L. Primary biliary cirrhosis: clinical features and outcome with special reference to asymptomatic disease. Scand J Gastroenterol 1989;24:57-64

5 Clarke AK, Galbraith RM, Hamilton EBD, Williams R. Rheumatic disorders in primary biliary cirrhosis. Ann Rheum Dis $1978 ; 37: 42-7$.

6 Uddenfeldt P, Bjerle P, Danielsson A. Evaluation of rheumatic disorders in patients with primary biliary cirrhosis. Ann Clin Res 1986;18:148-53.

7 Modena V, Marengo C, Amoroso A, Rosina F, Costantini P, Bellando P, et al. Primary biliary cirrhosis and rheumatic disease: a clinical, immunological and immunogenetical disease: a clinical, immunological and im
study. Clin Exp Rheumatol 1986;4:129-34.

study. Clin Exp Rheumatol 1986;4:129-34.
8 Kaplan MM. Primary biliary cirrhosis. N Engl J Med 1996; Kaplan MM. Prim

9 Subcommittee for Scleroderma Criteria of the American Rheumatism Association Diagnostic and Therapeutic Criteria Committee. Preliminary criteria for the classification of systemic sclerosis (scleroderma). Arthritis Rheum 1980; 23:581-90.

10 Tan EM, Cohen AS, Fries JF, Masi AT, McShane DJ, Rothfield NE, et al. The 1982 revised criteria for the classification of systemic lupus erythematosus. Arthritis Rheum 1982;25:1271-7.

11 Arnett FC, Edworthy SM, Bloch DA, McShane DJ, Fries $\mathrm{JF}$, Cooper NS, et al. The American Rheumatism Association 1987 revised criteria for the classification of rheumatoid arthritis. Arthritis Rheum 1988;31:315-24.

12 Vitali C, Bombardieri S, Moutsopoulos HM, Coll J, Gerli R, Hatron PY, et al. Assessment of the European classification criteria for Sjögren's syndrome. A series of clinically criteria for Sjogren's syndrome. A series of clinically defined cases: results of a prospecti

13 Sharp GC, Irving WS, May CM, Holman HR, McDuffie FC, Hess EV, et al. Association of antibodies to ribonucleoprotein and Sm antigens with mixed connective tissue disease, systemic lupus erythematosus and other rheumatic diseases. N Engl J Med 1976;295:1149-54.

14 Bohan A, Peter JB. Polymyositis and dermatomyositis. N Engl J Med 1975;292:344-7.

15 Seibold JR. Scleroderma and mixed connective tissue diseases. In: Ruddy S, Harris ED, Sledge CB, eds. Kelley's textbook of rheumatology. Philadelphia: WB Saunders, 2001: 1211-39.

16 Invernizzi P, Battezzati PM, Crosignani A, Zermiani P, Bignotto M, Del Papa N, et al. Antibody to carbonic anhydrase II is present in primary biliary cirrhosis (PBC) irrespective of antimitochondrial antibody status. Clin Exp Immunol 1998;114:448-54.

17 Myssor M, James OFW. The epidemiology of primary biliary cirrhosis in north-east England: an increasingly common disease? Q J Med (NS) 1990;78:377-85.

18 Parikh-Patel A, Gold E, Mackay IR, Gershwin ME. The geoepidemiology of primary biliary cirrhosis: contrasts and comparisons with the spectrum of autoimmune diseases. Clin Immunol 1999;91:206-18.

19 Medsger TA Jr. Epidemiology of systemic sclerosis. Clin Dermatol 1994;12:207-16.

20 Jacobson DL, Gange SJ, Rose NR, Graham NMH. Epidemiology and estimate of population burden of selected autoimmune diseases in the United States. Clin Immunol Immunopathol 1997;84:223-43.

21 Mayo MJ, Combes B, Jenkins RN. T cell receptor V $\beta$ gene utilization in primary biliary cirrhosis. Hepatology 1996; 24:1148-55.

22 Akimoto S, Ishikawa O, Takagi H, Miyachi Y. Immunological features of patients with primary biliary cirrhosis (PBC) overlapping systemic sclerosis: a comparison with patients overlapping systemic sclerosis: a comparison with patients 901 . 
23 Epstein O, Thomas HC, Sherlock S. Primary biliary cirrhosis in a dry gland syndrome with features of chronic graftsis in a dry gland syndrome with features

24 Siimes MA, Johansson E, Rapola J. Scleroderma-like graftversus-host disease as late consequence of bone marrow grafting. Lancet 1977; ii:831-2.

25 Sherer Y, Shoenfeld Y. Autoimmune diseases and autoimmunity post-bone marrow transplantation. Bone Marrow Trans 1998;22:873-81.

26 Artlett CM, Cox LA, Jimenez SA. Detection of cellular microchimerism of male or female origin in systemic sclerosis patients by polymerase chain reaction analysis of HLA-Cw antigens. Arthritis Rheum 2000;43:1062-7.

27 Klippel JH. Systemic lupus erythematosus: demographic prognosis and outcome. J Rheumatol 1997;24(suppl 48):67-71

28 Targoff IN. Polymyositis and dermatomyositis in adults. In: Maddison PJ, Isenberg DA, Woo P, Glass DN, eds. Oxford textbook of rheumatology. Volume 2. 2nd edn. Oxford: Oxford textbook of rheumatology. Volume 2.

29 Maddison PJ. Mixed connective tissue disease, overlap syndromes and eosinophilic fasciitis. Ann Rheum Dis dromes and eosin
30 Blas R, Rios C, Caraccio A, Hourclé S, Bagnati A, Ferrari Cifani $\mathrm{A}$, et al. Association of primary biliary cirrhosis and Sjögren's syndrome. Clin Exp Rheumatol 2000;18: 167.

31 Silman AJ. Epidemiology and the rheumatic diseases. In: Maddison PJ, Isenberg DA, Woo P, Glass DN, eds. Oxford textbook of rheumatology. Volume 2. 2nd edn. Oxford: Oxford University Press, 1998: 811-28.

32 Cimmino MA, Parisi M, Moggiana G, Mele GS, Accardo S. Prevalence of rheumatoid arthritis in Italy: the Chiavari study. Ann Rheum Dis 1998;57:315-18.

33 Leon-Perez L, Tiliakos NA. Digital vasculitis associated with centromere antibodies. Arthritis Rheum 1986;29: S89.

34 Marasini B, Pipia C, DeValle G, Crosignani A, Petroni L, Cugno $M$, et al. Vascular impairment in patients with primary biliary cirrhosis. Int J Microcirc 1995;5:75-9.

35 Belch JJF. Raynaud's phenomenon. Curr Opin Rheumatol 1989;2:937-41.

36 Walsh SJ, Pau LM. Autoimmune disease: a leading cause of death among young and middle-aged women in the United States. Am J Public Health 2000;90:1463-6. 